\title{
Auditory Feedback in Music Performance: Evidence for a Dissociation of Sequencing and Timing
}

\author{
Peter Q. Pfordresher \\ Ohio State University
}

\begin{abstract}
Four experiments examined temporal relationships between actions and auditory feedback in music performance. Experiment 1 incorporated phase shifts of feedback, which disrupted produced timing but not overall accuracy. Experiment 2 incorporated period shifts of pitch contents for synchronized feedback that primarily disrupted accuracy more than timing. Experiment 3 incorporated combined phase and period shifts, which caused moderate disruption to timing and accuracy and revealed interactive effects of period and phase shifts on production. A 4th experiment included all feedback conditions in the same session to confirm differences across Experiments 1-3. These results are consistent with the view that actions and their perceptual consequences are coordinated in a way that distinguishes timing (phase shifts) from sequencing (period shifts).
\end{abstract}

The production of complex sequences, such as music and speech, involves the coordination of motor actions that produce successive acoustic events. Given the cognitive and motoric complexity involved in such tasks, one can overlook how perceiving the results of one's actions affects production (cf. Hommel, Müsseler, Aschersleben, \& Prinz, 2001; Müsseler, 1999; Prinz, 1997). A particularly important issue in this context involves the kinds of congruencies between perception and action that are necessary to maintain the fluency of production. The current research addressed this issue by examining disruption of musical keyboard performances caused by varying the temporal relationships between produced actions (keypresses) and auditory events associated with these actions (auditory feedback). Furthermore, these experiments explored different levels of organization that may underlie the representation of complex sequences, such as melodies, by varying temporal relationships between actions and auditory feedback on different timescales.

Past investigations of sequence production with altered feedback in a variety of domains have led to two conclusions: Desynchronization of feedback affects the fluency of production, but alterations of feedback contents (e.g., pitches or phonemes) do not.

Peter Q. Pfordresher, Department of Psychology, Ohio State University.

This research was sponsored in part by National Institute of Mental Health Grant R01-45764 awarded to Caroline Palmer and by a James M. Klein Dissertation Proposal Award from the Ohio State University Department of Psychology. This work represents a portion of my doctoral dissertation, and I thank Caroline Palmer for invaluable guidance and support during this process. I also thank Mari Riess Jones, Bruno Repp, Richard Jagacinski, and Thomas Coyle for helpful comments on an earlier version of this article. Grant Baldwin, Annalisa Ventola, and David D. Luxton assisted with data collection, and Zebulon Highben assisted with stimulus construction. Thanks are also due to Steven Finney for generous technical assistance, including the creation of FTAP.

Correspondence concerning this article should be addressed to Peter Q. Pfordresher, who is now at the Department of Psychology and Institute for Music Research, University of Texas at San Antonio, 6900 North Loop 1604 West, San Antonio, Texas 78249-0652. E-mail: ppfordresher@ utsa.edu
Tasks using delayed auditory feedback (DAF), for instance, show disruption when a constant lag is added to auditory feedback onset times (this effect was first shown in speech by Black, 1951; Lee, 1950, 1951). DAF causes disruption such as slowing of production rate (for music: Finney, 1997; Gates, Bradshaw, \& Nettleton, 1974; for speech: Howell, 1983; Howell \& Archer, 1984; Howell \& Powell, 1987; Howell, Powell, \& Khan, 1983; for tapping: R. A Chase, Harvey, Standfast, Rapin, \& Sutton, 1961), increased errors (for music: Finney, 1997; for speech: Fairbanks \& Guttman, 1958; MacKay, 1968, 1970; Robinson, 1972; for tapping: Finney \& Warren, 2002), and increased timing variability (for music: Pfordresher \& Palmer, 2002). However, Finney (1997) found that randomization of feedback pitches did not disrupt piano performance, in contrast to significant disruption from DAF. Categorical alterations of content also do not appear to affect qualitative patterns of disruption from DAF, at least in speech production (Howell, 1983; Howell \& Archer, 1984; Howell et al., 1983; but see Finney, 1997, for an exception in music that is discussed later). The elimination of auditory feedback also does not appear to affect production in the context of music performance (Finney, 1997; Gates \& Bradshaw, 1974; Repp, 1999b), offering further support for the idea that alterations of feedback contents do not affect production.

An important limitation of this past research is that the kinds of manipulations used to vary feedback synchrony versus content have differed with respect to dependencies between actions and their consequences. Whereas timing characteristics of auditory feedback have depended on the timing of past actions (e.g., feedback onset time $=$ produced onset time $+\operatorname{Lag} x$ ), categorical alterations to feedback contents have usually been administered independently of produced contents (e.g., feedback pitches selected at random). It is possible that alterations to feedback contents may prove as disruptive as alterations of feedback synchrony when altered contents depend on past produced events. Such an effect would show that individuals are sensitive to relationships between altered auditory feedback and actions. One way to achieve this parity, adopted in the current study, is to alter feedback contents by having participants hear the auditory event associated 
with a past action in synchrony with the current action. Such a manipulation in music performance would involve delaying feedback on the timescale defined by the serial execution of pitch events. A recent speech production study manipulated auditory feedback timing in a similar way (Müller, Aschersleben, Esser, \& Müsseler, 2000). Speakers produced phonemes in synchrony with metronome pulses while auditory feedback was delayed by fixed time lags equal to the metronome period. Speakers who synchronized accurately would therefore hear feedback contents associated with the most recently produced phoneme $(i-1)$ in synchrony with the present produced phoneme $(i)$. The authors found some evidence for disruption in these conditions, which suggests that Lag 1 shifts of feedback contents may disrupt production. However, because speech timing is variable, the fixed time delays in Müller et al. (2000) would have resulted in asynchronies between motor actions and the onset times of auditory feedback. Disruptive effects of feedback asynchrony as well as contents may therefore have influenced results. Serial lags of feedback contents were manipulated in a more controlled way and over a broader span of past produced events in this article.

The current study modifies the feedback-alteration paradigm used in past research by adopting the constructs of period and phase in manipulations of auditory feedback timing. The distinction between period and phase is important in research on rhythmic behaviors (e.g., Jones, 1976; Kelso, 1995; Large, Fink, \& Kelso, 2002; Large \& Jones, 1999; Large \& Palmer, 2002; Pikovsky, Rosenblum, \& Kurths, 2001; Pressing, 1999; Repp, 2003; Semjen, Vorberg, \& Schulze, 1998; Thaut, Miller, \& Schauer, 1998), but it has not played any role in research concerning auditory feedback, despite the fact that some researchers have focused on the role of perception-action rhythms in DAF disruption (Finney \& Warren, 2002; Howell et al., 1983; Pfordresher \& Palmer, 2002; Robinson, 1972). Although phase and period are often distinguished in theoretical models of rhythm perception and production, many models propose interactions between processes controlling phase and period (e.g., Kelso, 1995; Large \& Jones, 1999). Relative phase characterizes the relationships between two periodic processes. Phase is often represented as a proportion of cycle duration, and relative phase can be represented as the proportion of the cycle in one process at which the cycle of another process begins. Period refers to the duration of a given cycle. When musicians perform isochronous melodies, the time spans separating successive actions (e.g., keypresses on the piano) form a period, or interonset interval (IOI), and phase indexes different time points between two successive keypresses. The timing of altered auditory feedback can be characterized according to its relative phase (i.e., its relation to the keypresses). When this relative phase reaches 1 , one can speak of a period shift (by lag) with relative phase of 0 .

Figure 1 shows schematically the way in which phase and period shifts of auditory feedback were manipulated in the current study. Phase shifts of auditory feedback, used in Experiment 1, are shown in the top half of the figure: Feedback onsets occurred after a proportion of each IOI (based on timing of previous IOIs) had elapsed. Feedback onsets from the first produced event could fall at phase shifts of approximately .33, .50, or .66 within the first IOI, and the same shift would continue through the rest of the performance. This manipulation resembled those used in past DAF research (e.g., Lee, 1950); absolute time lags used in those studies would be equivalent to constant relative phase shifts if participants

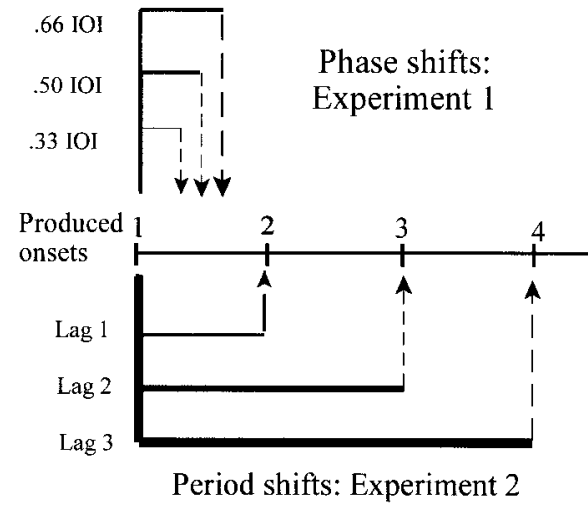

Figure 1. Examples of feedback manipulations in Experiments 1 and 2. Numbers 1-4 indicate successive isochronous produced onsets. Arrows indicate the timing of auditory feedback from the first produced event for different conditions. IOI $=$ interonset interval.

maintained a constant production rate. Moreover, phase shifts alter synchrony between actions and auditory feedback, but they do not alter the pitch contents in that performers hear the appropriate pitch following each keypress. Period shifts of auditory feedback, used in Experiment 2, are shown in the bottom half of Figure 1: The feedback pitch associated with the first produced keypress could coincide with the second, third, or fourth produced onset. This feedback alteration affected pitch contents (not synchrony) but did so differently than alterations used in past research, given that altered feedback contents depend on past produced events. Experiment 3 combined these manipulations: Feedback onsets were phase and period shifted. This manipulation (not shown in Figure 1) could result in the pitch produced at the first onset being heard between the second and third onsets. Experiment 4 included all three kinds of feedback manipulations to verify the combined results of Experiments 1-3.

These manipulations can be conceptualized as alterations of feedback along different hierarchical timescales. Disruption from each kind of alteration might differ qualitatively, insofar as the performer cognitively organizes a musical sequence in a way that distinguishes these timescales. Past research indicated time-based hierarchical organization of sequences in perception (e.g., Jones, 1976; Martin, 1972) and production (e.g., Collard \& Povel, 1982; Jagacinski, Marshburn, Klapp, \& Jones, 1988; Rosenbaum, Kenny, \& Derr, 1983). Furthermore, various research has suggested that timing and sequencing of successive events in production are guided by separate mechanisms (MacKay, 1987; Palmer \& Pfordresher, in press). The timescales at which timing and sequencing occur map onto phase and period, respectively. Functions that guide timing may therefore designate the time spans between successive events but not their contents (e.g., pitch identity, linguistic category). However, functions that guide sequencing may designate the contents of successive events, vis-à-vis categorical information, but not elapsed time, at least not in a fine-grained way.

The presumed separation between timing and sequencing may lead to a dissociation in disruption by auditory feedback manipulations. Phase shifts of auditory feedback, which alter feedback on the level of timing, may interfere with functions that guide timing 
but may not affect accuracy (i.e., the selection of responses mapped to appropriate contents). Conversely, period shifts of feedback, which alter feedback on the level of sequencing, may interfere with the selection of appropriate sequential content (thereby reducing accuracy) but may not affect timing. Experiment 3 provided a further way to test the effect of disruption when both the contents and synchrony of auditory feedback are altered through combined period and phase shifts. If the effect of each feedback alteration takes place at an independent level of organization (i.e., timing vs. sequencing), then phase and period shifts should yield independent effects on disruption. Comparisons across Experiments 1-3, as well as within Experiment 4, assessed whether these feedback alterations have an independent (additive) or interactive effect on production.

A final issue that the current study pursues is whether differences in planning of production can moderate the effect of auditory feedback. That is, disruption from feedback alterations may result in part from how they interact with the structure of plans. Plans are mental representations of a sequence that are used to retrieve events during production (Lashley, 1951; Norman, 1981). Early accounts of DAF disruption suggested that altered feedback disrupts planning because it registers as an error (e.g., R. A. Chase, 1965; Fairbanks, 1954; Fairbanks \& Guttman, 1958; Lee, 1950; but see Howell et al., 1983). For the purposes of the present study, the plan underlying production is considered to be a hierarchical representation in which the timescales relating to timing versus sequencing are separated. If disruption from altered feedback reflects discrepancies between feedback and planned events, then different planning strategies may modulate disruption. The first two experiments reported here included manipulations in which performers conceptualized performances differently at the level of timing (Experiment 1) or sequencing (Experiment 2).

\section{Experiment 1}

Pianists performed short, previously unfamiliar melodies from memory while listening to normal or phase-shifted auditory feedback. Phase-shifted tones occurred after their associated keypresses (i.e., were asynchronous) but before the next keypresses (see Figure 1, top). The phase shifts used were $.33, .50$, or .66 with respect to expected IOIs throughout a trial. It was predicted that these alterations would disrupt produced timing but not sequencing, resulting in increases of timing variability and slowing of production rate (relative to normal feedback conditions) but not increased error rates.

Subdividing instructions were also manipulated to test the hypothesis that disruption from feedback phase shifts results from discrepancies between feedback timing and planned timing. In different blocks of trials, performers were either given no subdividing instructions, instructions to mentally subdivide produced IOIs into two subintervals (.50 of planned IOIs), or instructions to mentally subdivide produced IOIs into three subintervals (.33 of planned IOIs). It was predicted that reduced disruption would occur when feedback onsets coincided with planned subdivisions. Therefore, when performers subdivided in 2, reduced disruption should have been found for the .50 phase shift relative to other instruction conditions. When performers subdivided in 3 , reduced disruption should have been found for the .33 and .66 phase shift conditions relative to other instruction conditions. This prediction was based on past research suggesting that mental subdivisions of time intervals underlie the cognitive representation of time and that produced timing is more accurate and precise when actions are synchronized with subdivisions (Semjen \& Ivry, 2001). Moreover, it was assumed that conceptualizing time intervals smaller than those performed would influence the planning of produced timing rather than sequencing. Some support for this prediction has been found in past research in which pianists performed repeated arpeggios at a prescribed rate with fixed feedback delays (Pfordresher \& Palmer, 2002). That study provided some evidence for reduced disruption when DAF onsets evenly divided produced IOIs, although an attempt to manipulate planning of subdivisions (as in the current Experiment 1) did not yield the predicted pattern of results. It was thought that the use of phase shifts rather than fixed delays might yield positive results.

\section{Method}

Participants. Twelve adult pianists (mean age $=21$ years, range $=$ 18-28) from the Columbus, Ohio, community participated in exchange for course credit in an introductory psychology class or payment. Pianists had 9.6 years of private piano training (range $=6.0-15.0$ ) and 11.4 years of experience playing the piano (range $=8.0-15.5$ ) on average. Ten reported being right-handed, and 2 reported being left-handed. Nine participants were female, and 3 were male.

Materials. Two melodies that were notated in a binary meter (2/4 time signature) served as stimulus materials. One melody was notated in the key of $\mathrm{G}$ major, the other in $\mathrm{C}$ major. In order to avoid the use of stereotyped motor movements, melodies did not contain repeating pitch patterns. Both melodies were performed with the right hand only. The top half of Figure 2 shows the stimuli used for Experiment 1.

\section{Stimuli for all Experiments: Binary Meters}

A:

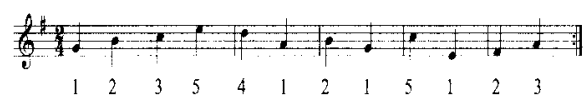

B:

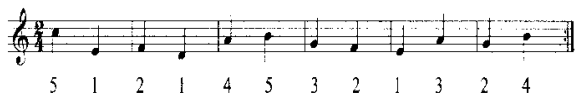

Additional Stimuli for Experiment 2: Ternary Meters

A:

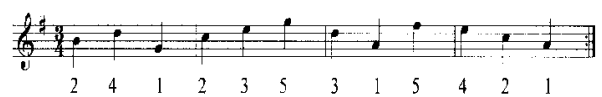

B:

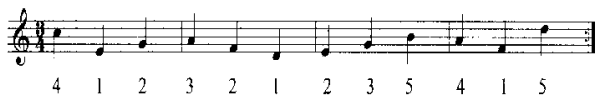

Figure 2. Stimuli (G major $[\mathrm{A}]$ and $\mathrm{C}$ major $[\mathrm{B}]$ melodies) used in all experiments and additional stimuli for Experiment 2, shown in music notation. Numbers beneath the notes indicate right-hand fingering $(1=$ thumb, 2 = index, etc.). 
Conditions and design. Experiment 1 included four feedback conditions: normal (immediate) feedback and auditory feedback phase shifts of $.33, .50$, and .66 . These were crossed with three subdividing conditions: no instructions, instructions to subdivide using two subintervals (subdivide-2), and instructions to subdivide using three subintervals (subdivide-3), creating a $4 \times 3$ within-participants design. Participants performed both melodies in each of the 12 conditions, yielding 24 experimental trials. Trials were blocked first by instruction condition, then by melody, yielding six blocks of four trials each, which were presented in a single session. The two no-instructions blocks always occurred first. The order of feedback conditions varied within each block, except that the immediate feedback condition always occurred first. The following additional factors were counterbalanced in a Latin square design that yielded four order conditions: order of the subdivide- 2 and subdivide- 3 instruction conditions, order of melody, blocks within instruction conditions, and two orders of feedback conditions.

Apparatus. Participants performed the melodies on a Roland RD-600 weighted-key digital piano, which simulated the feel of an acoustic piano. Presentation of auditory feedback and metronome pulses, as well as Musical Instrumental Digital Interface (MIDI) data acquisition, were all implemented by the FTAP software program (Finney, 2001). Participants heard performances and metronome pulses over AKG K270 headphones; the piano timbre originated from Program \#1 (Standard Concert Piano 1), and the metronome timbre originated from Program \#126 (Standard Set, MIDI Key $56=$ cowbell ) of the RD-600. MIDI velocity of auditory feedback was held constant; the sound intensity was approximately 80-dB sound pressure level (measured by a General Radio Model 1982 soundlevel meter centered at $1 \mathrm{kHz}$ with A weighting, coupled to a Telephonics TDH-39 audiometer earphone).

Procedure. At the beginning of a session, participants practiced the first melody with immediate feedback until it was memorized and performed without errors, after which the music notation was removed. Then participants performed at least two repetitions of that melody from memory in synchrony with the metronome, at the prescribed rate $(500 \mathrm{~ms}$ between metronome onsets). Then participants performed it with a .66 feedback phase shift (similar to the absolute time delay found to be most disruptive by Pfordresher \& Palmer, 2002) at a comfortable self-selected rate for another two repetitions to become accustomed to the experience of altered feedback. Following this familiarization with synchronization and altered auditory feedback, participants performed at least one practice trial using the .66 phase shift.

Each trial used a synchronization-continuation paradigm in which altered feedback conditions occurred during the continuation phase. In the synchronization phase, participants performed a melody with the metronome (500 ms between metronome onsets) and normal feedback. After 24 note onsets (two repetitions of the melody for error-free performances), the metronome stopped and the participant attempted to maintain that rate during the continuation phase under one of the auditory feedback conditions. The continuation phase lasted for another 104 keypresses (approximately eight repetitions of the melody), after which the cessation of feedback signaled the end of the trial. The time lag for each auditory feedback onset was a proportion of the expected IOI length, which was based on the average of the previous two IOIs (excluding any IOIs that were less than $30 \mathrm{~ms}$ or greater than $1 \mathrm{~s}){ }^{1}$

Each block included four experimental trials, one for each of the feedback conditions (normal feedback, .33, .50, and .66). The first block was performed with the same melody used for practice trials, with no subdividing instructions. Then participants practiced the second melody with immediate feedback, in view of the notation, and afterwards they completed a block of four trials for that melody with the notation removed and no subdividing instructions. Following a short break, participants performed the same two melodies during the third and fourth blocks while carrying out one of the subdividing instruction conditions. Participants then carried out the other subdividing instructions condition for each melody in the two final blocks. Because each new block of trials resulted in changing from one melody to the other, participants performed the new melody with immediate feedback and while looking at the music notation at the beginning of each block to refresh their memory. Participants completed a questionnaire concerning their musical experience before Block 5. At the end of the session, which lasted about $1 \mathrm{~h}$, participants completed a postexperiment questionnaire concerning the task.

Before Blocks 3 and 5, the experimenter gave relevant subdividing instructions. Subdividing was described as "counting in your head at a rate faster than the rate of your performance in a way that evenly divides the durations you produce." An auditory example of subdivision timing relative to produced timing was also provided by presenting two metronome pulse trains: a louder train that marked the rate of production $(500 \mathrm{~ms}$ between metronome onsets) was sounded first and then combined with a quieter pulse train that marked the rate of subdivisions $(250 \mathrm{~ms}$ or $167 \mathrm{~ms}$ between metronome onsets). During this example, the experimenter verbalized the sort of cyclical mental counting that a participant might use, which is standard in music pedagogy: "one-two, one-two ..." for binary subdivisions and "one-two-three, one-two-three ..." for ternary subdivisions in synchrony with the quieter pulse train. Participants were instructed to maintain a consistent tempo throughout each trial and to avoid correcting any errors.

Data analyses. Analyses of each trial excluded the entire synchronization phase, the first repetition of the melody in the continuation phase, and any events following the first produced event of the eighth repetition in the continuation phase. This resulted in six repetitions of the melody followed by a repetition of its first event $(73$ sequence events $=72$ IOIs in performances without additions or deletions) that were analyzed for each trial.

Error rates (number of incorrectly produced pitches/73 sequence events) indexed accuracy for each trial. Errors were detected using software that compared pitches produced in performances to those that would occur in a correct performance (Palmer \& van de Sande, 1993, 1995). Errors in the continuation phase that also occurred during the synchronization phase of the same trial were eliminated because such errors might reflect learning or memory problems unrelated to feedback alterations. Errors that were filtered out in this way made up a small percentage of total errors in each experiment $(4.0 \%, 8.0 \%, 0.1 \%$, and $1.0 \%$ in Experiments $1-4$, respectively).

Variability and means of produced IOIs indexed disruption of produced timing; variability was measured using coefficients of variation (CVs = standard deviation of IOI/mean IOI within a trial). Both measures of produced timing incorporated adjustments to remove potentially spurious effects of errors and tempo drift. First, each repetition of the sequence was divided into two halves (Notes 1-6 or 7-12 shown in Figure 2), and when pitch errors occurred, the error and the half-sequence surrounding it were removed (cf. Meyer \& Palmer, 2002; Palmer \& Drake, 1997). This practice was based on evidence that errors in piano performance are constrained by higher-order conceptual units, such as phrases (Palmer \& van de Sande, 1995); the two halves of each melody may be considered as constituting phrases. Following error removal, outliers were removed (defined as plus or minus three standard deviations around mean IOI per trial), and the

\footnotetext{
${ }^{1}$ Pilot studies indicated that using two previous IOIs maintained the most consistent IOI-feedback timing relationships in conditions in which produced timing fluctuated compared with one or four preceding IOIs. The average of three preceding IOIs was not considered due to the binary meter of the stimuli.
} 
remaining IOIs in a trial were detrended by adding the mean IOI to the residual IOI values from a linear regression of IOI on sequence position. ${ }^{2}$

\section{Results}

The effects of feedback phase shifts and subdividing instructions were tested in two repeated measures analyses of variance (ANOVAs) for each dependent variable. Results for each dependent variable are shown in Figure 3. The first ANOVA for each measure used a 2 (feedback type $=$ normal, altered) $\times 3$ (instruction condition $=$ none, subdivide- 2 , subdivide-3) design, which assessed overall disruption from altered feedback. The second ANOVA assessed disruption across phase shifts and their interaction with instructions with a 3 (phase shift $=.33, .50, .66) \times 3$ (instruction condition) design. To control for possible violations of sphericity, I applied Geisser-Greenhouse corrections to all repeated measures ANOVAs with more than one degree of freedom in the numerator. Reported $p$ values reflect this correction, where applicable, although the correction did not change statistical significance (critical $\alpha=.05$ ) for any results of theoretical importance.

Figure 3A shows mean CVs by feedback and instruction condition. The first ANOVA demonstrated that altered feedback increased timing variability, $F(1,11)=70.27, M S E=.001, p<.01$, and subdividing instructions decreased timing variability, $F(2$, $22)=9.31, M S E=.002, p<.01$, overall. $\mathrm{CV}$ s also increased with increasing phase shifts, $F(2,22)=35.96, M S E=.003, p<.01$,

A

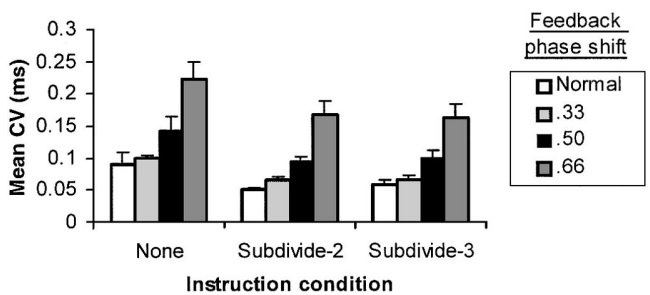

B
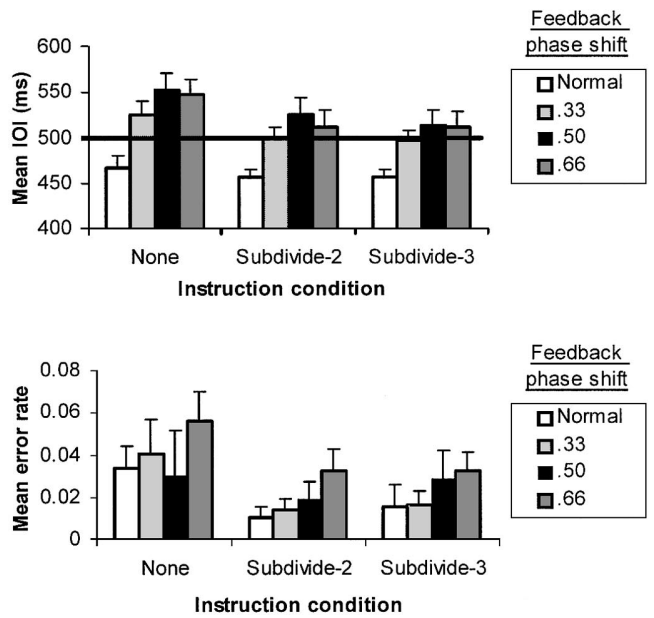

Figure 3. Disruptive effects of feedback alterations in Experiment 1 as indexed by (A) mean coefficients of variation (CVs: standard deviation of interonset interval $[\mathrm{IOI}] /$ mean IOI), (B) mean IOIs (black line corresponds to the $500 \mathrm{~ms}$ between metronome onsets), and (C) mean error rates. Error bars represent one between-participants standard error. demonstrated in the second ANOVA. No Feedback Type $\times$ Instruction Condition interactions were found. In particular, the predicted interaction in the second ANOVA, reflecting less disruption at the .50 phase shift for subdivide- 2 and less disruption for the .33 and .66 phase shifts for subdivide-3, was not obtained.

Results for mean IOIs, the second measure of timing disruption, are shown in Figure 3B. Performances were slower with altered feedback than with normal feedback, $F(1,11)=38.00, M S E=$ $1,724, p<.01$, as demonstrated in the first ANOVA. Performances with normal feedback were faster than the prescribed tempo on average [mean IOI $=458.80 \mathrm{~ms}, t(71)=-8.19, p<$ .01] and were slower than the prescribed tempo under altered feedback conditions [mean IOI $=520.13 \mathrm{~ms}, t(216)=4.56, p<$ .01]. Unlike CV measures, the effects of subdividing instructions were qualified by an interaction with feedback condition in the first ANOVA, $F(2,22)=3.98, M S E=282, p<.05$. Performances with altered feedback were faster when performers subdivided than when they did not subdivide, but subdividing did not affect performances with normal feedback. The second ANOVA failed to reveal any differences across different feedback phase shifts, and no Phase Shift $\times$ Instruction Condition interactions emerged.

Mean error rates are shown in Figure 3C. Altered feedback did not reliably increase error rates overall. The main effect of feedback type on error rates in the first ANOVA did not reach significance $(p=.09)$. Subdividing instructions did reduce error rates, however, $F(2,22)=7.24, \operatorname{MSE}<.001, p<.01$. Although phase shifts did not significantly increase error rates overall, relative to normal feedback, error rates did increase across different phase shift amounts, with the most errors being made for the .66 shift, $F(2,22)=4.79, M S E=.001, p<.05$.

The possibility that phase shifts disrupted timing but not accuracy was further examined through effect sizes. Partial $\omega^{2}$ s for within-participants factorial designs (Kirk, 1995, p. 459) were estimated from the main effect of feedback type in the first ANOVAs for each dependent variable (CVs, IOIs, error rates). The estimated $\omega^{2}$ was higher for CVs (.59) and IOIs (.34) than for error rates (.04). It should be noted that the value of $\omega^{2}$ that indicates a "large" effect size (.15) lies between estimates for timing measures and that for error rates (Keppel, 1991). This result corroborates differences in levels of significance for main effects of feedback type in these ANOVAs.

Results for CVs and error rates both revealed facilitation from subdividing instructions for normal and altered feedback conditions. It is possible that these differences might have resulted from practice effects, because the no-instructions blocks were always

\footnotetext{
${ }^{2}$ Removal of half-sequences surrounding errors and removal of outliers had a substantial effect on overall variability in Experiment 1 (mean CV = .2 before these procedures; mean $\mathrm{CV}=.12$ afterward), despite the fact that these procedures resulted in the removal of relatively few produced events per trial (a reduction of 6.78 out of 73 on average, or 9\%). Adjustments of IOIs for linear tempo drift had a smaller effect on overall variability (mean $\mathrm{CV}=.11$ after adjustments). However, removal of drift appeared to be appropriate given the presence of a significant linear relationship between produced event number and IOI before IOIs were adjusted for tempo drift (mean $r=.24, p<.01$ ). The same procedures yielded generally similar effects in other experiments, with the exception that more events were removed based on errors and outliers in Experiment 2 ( $M=22$ events).
} 
first. This possibility was investigated by examining changes to $\mathrm{CVs}$ and error rates across the six successive blocks in a session. Although the effect of block number on CVs was significant, $F(5$, $55)=7.79$, MSE $=.001, p<.01$, the pattern of results did not reflect a gradual improvement with practice. Post hoc tests (Tukey's honestly significant difference [HSD]) revealed higher CVs for Block $2(M=.1504)$ than Block $1(M=.1277)$. More important, lowest $\mathrm{CV}$ s were found in Blocks 3-6 $(M=.0950$ across blocks), for which subdividing instructions were used, and none of these later blocks differed from each other. It therefore appears that subdividing instructions, rather than a general practice effect, accounts for the lower CVs found in blocks that included instructions. A similar pattern of results was found for error rates, although the effect of block fell short of significance $(p=.07)$. Because effects of instructions on mean IOIs were limited to altered feedback conditions, the effect of practice on mean IOIs was not examined.

\section{Discussion}

Experiment 1 yielded three main findings. First, phase shifts of auditory feedback primarily disrupted timing, with no overall effect on accuracy. Feedback alterations significantly increased timing variability $(\mathrm{CVs})$ and slowed production rate but did not significantly increase error rates overall in comparison with normal feedback conditions, although error rates did vary with phase shift amount. ${ }^{3}$ This dissociation in statistical significance was bolstered by effect sizes, which revealed a larger disruptive effect of altered feedback on timing (CVs, IOIs) than on accuracy (error rates). Second, increases with phase shift amount were found for both CVs and error rates, although disruption reached an asymptote at the .33 phase shift for mean IOIs. Finally, subdividing instructions (binary or ternary) benefitted performance, but the pattern of responses did not support the idea that discrepancies between feedback timing and planned timing accounted for disruption from feedback phase shifts. The benefit of subdividing instructions was not specific to coincidences between feedback onsets and planned subdivisions. Instead, subdividing instructions reduced disruption across all altered feedback conditions.

These results are consistent with the idea that feedback phase shifts disrupt timing more so than sequencing. However, because some disruption of accuracy was evident in error rates, it was necessary to show that alterations of feedback contents cause the reverse effect: disruption of accuracy rather than timing. Experiment 2 was designed to test this corollary prediction.

\section{Experiment 2}

Experiment 2 introduced period shifts of auditory feedback. Auditory feedback onsets occurred synchronously with produced keypresses, but feedback pitches were altered to match pitches associated with earlier keypresses by a lag of one, two, or three events (see Figure 1). Some past research has implied that alterations to the contents of auditory feedback do not disrupt production, as discussed earlier. However, the alterations used here differed from those used previously in that the pitch contents of altered feedback resulted from past actions. Period shifts of feedback were predicted to disrupt sequencing but not timing because these alterations affect the relationship between produced and perceived contents but not synchrony. This prediction was based on past work suggesting that sequencing mechanisms guide the selection of produced contents by using categorical information but not the time spans between successive produced actions (MacKay, 1987; Palmer \& Pfordresher, in press). Operationally, error rates, but not CVs or IOIs, were predicted to increase with period shifts of feedback. ${ }^{4}$

The hypothesis that period shifts conflict with the planning of sequencing was also explored by varying the notated meter of performed stimuli. Musical meter categorizes successive beats in a melody according to their degree of accentuation (stronger vs. weaker); strong and weak beats alternate to establish a higherorder periodicity (usually binary or ternary; e.g., Cooper \& Meyer, 1960; Lerdahl \& Jackendoff, 1983; see Liberman \& Prince, 1977, for a discussion of similar meters underlying language production). Unlike other musical accents (e.g., Jones \& Pfordresher, 1997; Pfordresher, 2003), metrical accents do not necessarily result from changes in the acoustic signal (Drake \& Palmer, 1993), resulting instead from inferences made by the listener or performer. Recent evidence suggests that conceptualized metrical accents may guide retrieval during production such that several events of similar accent strengths are mentally accessible at a given point in time (Palmer \& Pfordresher, in press; Palmer \& Schendel, 2002; cf. Lashley, 1951; Norman, 1981). A large amount of evidence also indicates that meter guides the perception of and memory for music (e.g., Large, 2000; Palmer \& Krumhansl, 1990; Povel, 1981; Povel \& Essens, 1985) and may also guide language perception (e.g., Pitt \& Samuel, 1990).

If perception and action use a shared representation, it is possible that performers use metrical relationships among events to plan produced actions and are also sensitive to metrical accents in co-occurring auditory feedback. Specifically, disruption should be greater when metrical accents associated with feedback events conflict with the metrical accents of events that are accessible in planning. ${ }^{5}$ Metrical similarity in the current stimuli is maximal for events separated by serial lags of two in binary meters and by

\footnotetext{
${ }^{3}$ Increases in mean IOI were considered as indicating disruption based on the conclusions of previous studies that manipulated DAF (e.g., Gates et al., 1974; Howell et al., 1983), despite the fact that production was closer to the target tempo in some altered feedback conditions relative to normal feedback conditions. Obtained findings for other dependent measures, which clearly show disruption, support this assumption.

${ }^{4}$ Although it is common to analyze sequencing mechanisms through errors, past studies have often restricted such analyses to errors with an identifiable source in the sequence (e.g., Bock, 1995, 1999; Dell, 1986; Fromkin, 1973; Garrett, 1980; Palmer \& van de Sande, 1993, 1995). These errors, on their own, suggest rule-based processes of sequencing (Bock, 1999). However, it is possible that errors omitted from these analyses also resulted from sequencing mechanisms (Dell, 1986). Moreover, constraints on the types of errors used for analysis are necessary when drawing conclusions about sequencing mechanisms based only on error types (the consequences of planning). By contrast, Experiment 2 manipulated presumed causes for disruption to sequencing mechanisms. Therefore, the rate of occurrence for all error types (except those that also occurred during the initial synchronization phase) for altered feedback conditions was interpreted as reflecting disruptions of sequencing in the current study.

${ }^{5}$ Although planning is often future based, past and current events also appear to be highly accessible within a plan at any given point in time (e.g., Dell, Burger, \& Svec, 1997; Palmer \& Pfordresher, in press). Moreover,
} 
serial lags of three in ternary meters. Therefore, binary meter performances should be less disrupted by Lag 2 shifts relative to Lag 3 shifts, and the reverse should be found with ternary meters.

\section{Method}

Participants. Twenty-one adult pianists (mean age $=21$ years, range $=19-32$ ) from the Columbus, Ohio, community who had not participated in Experiment 1 participated for course credit in an introductory psychology class or payment. Pianists had 10.0 years of private piano training (range $=6.0-14.0$ ) and 13.4 years of experience playing the piano (range $=10.0-22.0)$ on average. Two pianists reported being left-handed, and 1 reported being ambidextrous. Sixteen participants were female, and 5 were male.

Materials. Along with the melodies used in Experiment 1, two additional melodies were used in Experiment 2, and these were notated in a ternary meter (3/4 time signature). These new melodies are shown in the bottom half of Figure 2. Aside from the meter, the melodies were structured in similar ways to those used in Experiment 1. One of the new melodies was notated in the key of $\mathrm{G}$ major, and the other was in $\mathrm{C}$ major. All four melodies were constrained such that no pitch could repeat within a span of three notes, which prevented coincidences of planned and feedback pitches during period shift conditions.

Conditions and design. The four feedback conditions included normal (immediate) feedback and period shifts of Lags 1,2, and 3. These were crossed with two meter conditions: binary ( $2 / 4$ notated time signature) and ternary (3/4 notated time signature) to yield a $4 \times 2$ within-participants design. Each meter condition was linked to a single melody for a given participant (i.e., each performed only two of the four stimulus melodies; melodies were assigned randomly to participants). Participants performed two repetitions of each Meter $\times$ Feedback condition to yield 16 trials in a session. Trials were blocked by meter, and the order of feedback conditions varied across blocks. Participants experienced all four feedback conditions in a block before the second repetition of each feedback condition. Counterbalancing of order variables (order of meter, order of stimulus, order of trials within blocks) was carried out as in Experiment 1 to yield four order conditions (Latin square design); normal feedback trials were always the first and last trials in a block.

Procedure. The procedure on each trial for this experiment was identical to that in Experiment 1. The FTAP program (Finney, 2001) played the pitch produced $n$ events previously (where $n=0$ for normal feedback; $n=$ 1,2 , or 3 for altered feedback conditions) at each keypress. Participants therefore heard previously produced errors as well as correct events. A brief break occurred between the two blocks, during which participants completed a questionnaire regarding musical experience. Practice and instructions were carried out as in Experiment 1, except that here no subdividing instructions were given.

\section{Results}

Mean CVs, IOIs, and pitch error rates for Experiment 2 were examined as in Experiment 1, and these are shown in Figure 4. Two ANOVAs were run for each dependent variable: The first tested overall disruption from period shifts with a 2 (feedback type $=$ normal, altered $) \times 2$ (meter $=$ binary, ternary) design, and

given that the strength of accessibility for future events within a plan takes time (MacKay, 1987), it is likely that the most salient event in planning when auditory feedback is heard would be the current event. Therefore, it is assumed here that similarity-based interference is calculated relative to the current, rather than the next, event.
$\mathbf{A}$

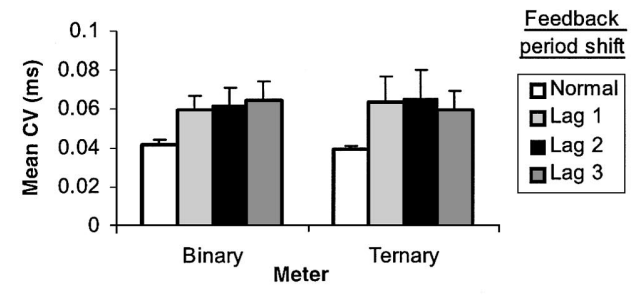

B

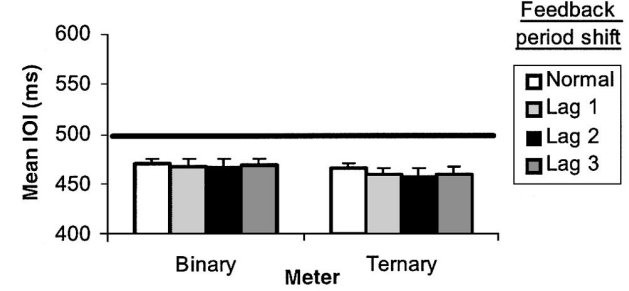

$\mathbf{C}$

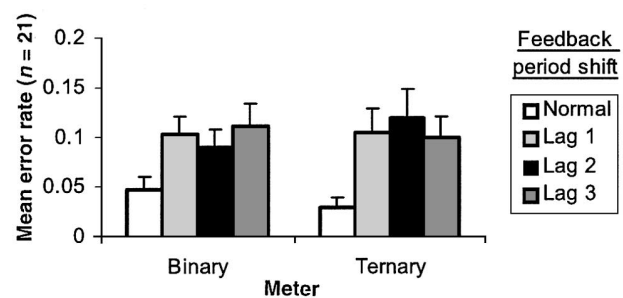

D

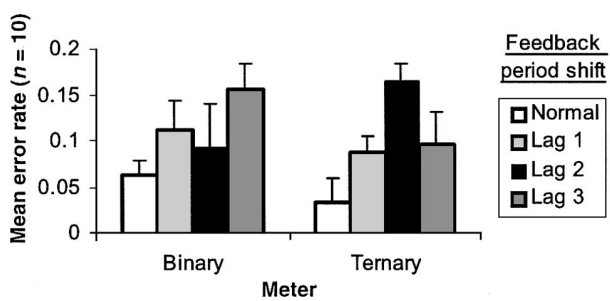

Figure 4. Disruptive effects of feedback alterations in Experiment 2 as indexed by (A) mean coefficients of variation (CVs: standard deviation of interonset interval [IOI]/mean IOI), (B) mean IOIs (black line corresponds to the $500 \mathrm{~ms}$ between metronome onsets), and (C) mean error rates for all participants. D: Mean error rates for 10 participants whose individual data qualitatively reflect the predicted interaction of meter and period shift amount. Error bars represent one between-participants standard error.

the second tested effects across different period shifts with a 3 $($ period shift $=\operatorname{Lag} 1,2$, or 3$) \times 2$ (meter) design

Mean error rates, which were predicted to be increased by period shifts, are shown in Figure 4C for all participants. Altered feedback increased error rates for both meters, $F(1,20)=15.40$, $M S E=.006, p<.01$, shown in the first ANOVA. Although there was a tendency toward the predicted Meter $\times$ Period Shift interaction, these differences did not yield a significant interaction in the second ANOVA, which also yielded no main effects (error rates did not increase with magnitude of period shifts). A subsequent ANOVA verified that increases in error rates were greater for Experiment 2 than Experiment 1 through a significant Experiment $(1,2) \times$ Feedback Type (normal, altered) interaction, $F(1$, 31) $=5.96$, MSE $=.002, p<.05$.

Examination of individual participant data suggested that the data in Figure 4C reflect a composite distribution of participants who did or did not show the predicted interaction (higher disrup- 
tion at Lag 3 than at Lag 2 for binary meters, and the opposite for ternary meters). The mean data for a subgroup of 10 participants are shown in Figure 4D. This subgroup showed not only disruption from altered feedback, as did the entire group, $F(1,9)=8.10$, $M S E=.006, p<.05$, but also the predicted Meter $\times$ Period Shift interaction, $F(2,18)=9.04, M S E=.003, p<.01$, in the second ANOVA.

Disruption to timing, which was not predicted to increase from period shifts, was indexed by $\mathrm{CVs}$ and mean IOIs. Figure 4A shows mean $\mathrm{CVs}$ for all participants. Contrary to predictions, altered feedback significantly increased CVs, evidenced by the main effect of feedback type in the first ANOVA, $F(1,20)=8.58$, $M S E=.001, p<.01$. The second ANOVA revealed no significant effects. Results for mean IOIs, the second measure of timing disruption, are shown in Figure 4B. Unlike with CVs, and consistent with predictions, no influence of altered feedback on mean IOIs was evident $(p=.13)$. No main effects or interactions were found in either ANOVA. As in Experiment 1, performances were faster than the prescribed rate of 500-ms IOIs across both normal and altered feedback conditions [mean performed IOI $=464.80$ ms, $t(335)=-13.69, p<.01]$.

The prediction that accuracy would be more disrupted than timing precision in Experiment 2 was further examined through effect sizes, as in Experiment 1. Although significant effects of altered feedback were found for both error rates and CVs, partial $\omega^{2} \mathrm{~s}$ indicated a larger effect for the former than the latter $\left(\omega^{2}\right.$ for errors $=.15$; for $\mathrm{CVs}=.08)$. Note that a "large" effect size (.15) was obtained for error rates, whereas the effect size for CVs was closer to a "medium" effect size (.06). Not surprisingly, the effect size for mean IOI was small (.02).

\section{Discussion}

The results of Experiment 2, in combination with those of Experiment 1, support the predicted double dissociation: Period shifts of feedback in Experiment 2 disrupted accuracy more than timing, whereas phase shifts of feedback in Experiment 1 disrupted timing but not accuracy. Moreover, Experiment 2 demonstrated disruption when pitch contents of auditory feedback were altered to match past produced events, which contrasts with past research, in which performance was unaffected by manipulations of feedback contents (Finney, 1997; Howell, 1983; Howell \& Archer, 1984; Howell \& Powell, 1987; Howell et al., 1983). Although other methodological differences may account for discrepancies between the present and past results, Experiment 2 nevertheless provides new evidence that alterations of feedback contents alone can disrupt performance. Finally, about half of the participants showed the predicted interaction between meter and period shift, which supports the idea that, for them, disruption in Experiment 2 resulted from discrepancies between feedback and sequence planning.

Although the error rates found in Experiment 2 appear low (normal feedback mean $=.050$; altered feedback mean $=.100$ ), they are similar to those found in other studies that have examined errors in skilled piano performance. Palmer and Drake (1997) reported an error rate of .03 for a group of performers with similar amounts of experience (mean years of training $=10$ ) performing more complicated but familiar melodies. Another recent study (Palmer \& Pfordresher, in press) reported error rates somewhat closer to the current study's altered feedback condition (.080) for rapid performances of purposefully complex melodies. Despite these low error rates, which are characteristic of skilled musical performance, patterns of errors proved diagnostic of mechanisms underlying production, insofar as these differences are statistically reliable and connected theoretically to underlying mechanisms.

The results of Experiments 1 and 2, taken together, support the idea that phase shifts disrupt timing, whereas period shifts disrupt sequencing. However, the fact that some disruption to both timing and sequencing was evident in each experiment suggests that these effects are not independent. Experiment 3 further addressed the possibility that period and phase shifts are independent by examining performances with feedback that was both phase and period shifted.

\section{Experiment 3}

Experiment 3 investigated combined period and phase shifts of auditory feedback. In altered feedback conditions, pitches matched earlier produced pitches, but feedback onsets were desynchronized from produced onsets. Different combinations of period and phase shifts were treated as separate variables in statistical analyses, rather than as a single continuum, in order to examine the degree to which these alterations yielded independent or interactive effects. The same stimulus materials were used as in Experiment 1, and the instructions and procedure were implemented as in Experiment 2 .

\section{Method}

Participants. Fourteen adult pianists (mean age $=24$ years, range $=$ 18-51) from the Columbus, Ohio, community participated in this experiment for course credit in an introductory psychology class or payment. None had participated in either of the first two experiments. Pianists had 9.1 years of private piano training (range $=2.5-13.0$ ) and 16.3 years of experience playing the piano (range $=5.0-44.0$ ) on average. One participant was retained despite having very few years of private lessons (2.5) because of many years' experience playing the piano (27.0). Twelve pianists reported being right-handed, and the remainder reported being left-handed. Eight participants were female, and 6 were male.

Conditions and design. Three levels of feedback phase shift (.33, .50, and .66) were crossed with three levels of feedback period shift (Lag 1, 2, and 3 ) to yield a $3 \times 3$ within-participant design. A normal (immediate) feedback condition was also included for purposes of comparison, yielding 10 feedback conditions. Participants performed each stimulus melody in all 10 conditions, yielding 20 trials in a session. Trials were blocked by stimulus melody according to two different orders. The normal feedback trial initiated each block, and the remaining experimental conditions were randomized in two different orders, which yielded four different order conditions, as in the previous experiments.

\section{Results}

Differences between the two normal feedback trials and all altered feedback trials combined were assessed in a one-tailed $t$ test. A two-way ANOVA then examined different combinations of alterations with the variables phase shift $(.33, .50, .66)$ and period shift (Lags 1, 2, and 3). Mean data for all dependent measures are shown in Figure 5.

Mean error rates, shown in Figure 5C, increased significantly with the introduction of altered feedback, $t(13)=3.28, p<.01$, as 
A

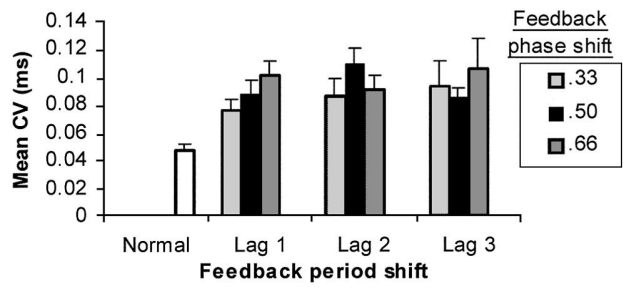

B

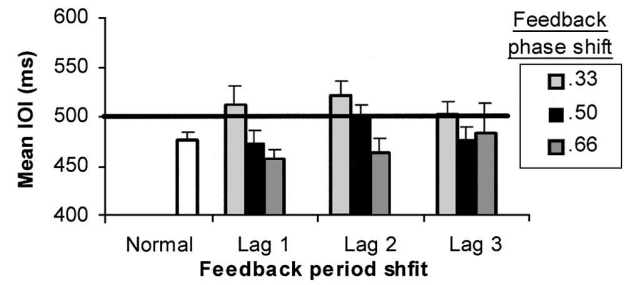

C

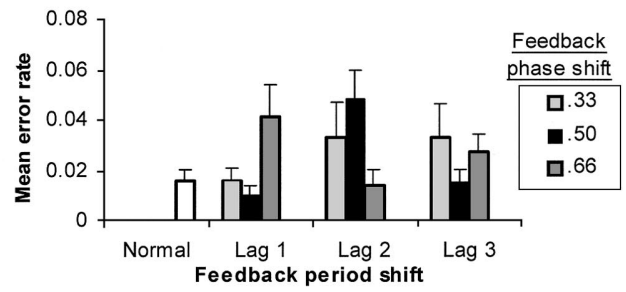

Figure 5. Disruptive effects of feedback alterations in Experiment 3 as indexed by (A) mean coefficients of variation (CVs: standard deviation of interonset interval [IOI]/mean IOI), (B) mean IOIs (black line corresponds to the $500 \mathrm{~ms}$ between metronome onsets), and (C) mean error rates. Error bars represent one between-participants standard error.

in Experiment 2. The ANOVA revealed a significant Phase Shift $\times$ Period Shift interaction, $F(4,52)=4.76, M S E=.001$, $p<.01$. The only significant pairwise difference was between the .50 phase shifts at Lags 1 and 2 (Tukey's HSD, $p<.05$ ). No main effect of either feedback alteration was found, indicating that no overall differences across different phase or period shifts occurred. However, the results for Lag 1 were in good agreement with those of Experiment 1 (Figure 3). The qualitative pattern of error rates across period shifts differed from that found in Experiment 2, with higher error rates overall at Lag 2 (.032) than at other lags (Lag $1=.022$, Lag $3=.025)$, contrary to what might be expected on the basis of the binary meter of the melodies.

Figure 5A plots mean $\mathrm{CV}$ across all combinations of phase and period shifts, along with the baseline condition. Altered feedback increased CVs relative to normal feedback, as revealed by a significant $t$ test comparison, $t(13)=5.32, p<.01$. The interaction between types of alterations fell short of significance $(p=.12)$, although the overall pattern of results was similar to that found for error rates $(r=.79$ between results for CVs and error rates, $p<$ $.01)$. As with error rates, no main effect of either feedback alteration emerged in the ANOVA.

Mean IOIs were also examined, and these revealed a different pattern of results than did CVs and error rates, as shown in Figure 5B. Combined alterations did not slow down production rate overall $(F<1)$. Also, no Period Shift $\times$ Phase Shift interaction emerged in the ANOVA. However, a main effect of phase shift was found, $F(2,26)=13.27, M S E=1,597, p<.01$, which reflected an increase in production rate with increasing feedback asynchrony. The results for .33 phase lag resemble those of Experiment 1 (Figure 3), showing a decrease in production rate relative to the normal feedback condition. The results for other phase shifts are more similar to those of Experiment 2 (Figure 4), in which no differences were obtained.

As in Experiments 1 and 2, effect sizes for the introduction of altered feedback, compared with normal feedback, were used to assess whether combined delays primarily disrupted timing or accuracy. Results were qualitatively similar to those found for Experiment 1. The main effect of feedback type on CVs yielded a larger partial $\omega^{2}(.50)$ than the main effect of feedback condition on error rates (.20), with both values indicating "large" effect sizes. However, the effect size for mean IOI was the smallest $(-.01)$, which makes conclusions about disruption to timing versus accuracy within Experiment 3 difficult.

\section{Comparisons Across Experiments 1-3}

Analyses that compared results across experiments for each measure of disruption focused on difference scores between normal and altered feedback conditions. These analyses included conditions in different experiments that shared the same stimuli and instructions to participants: the no-instructions conditions of Experiment 1, the binary meter conditions of Experiment 2, and all conditions of Experiment 3. It was predicted that each index of disruption would reveal maximal increases for the experiment in which feedback alterations occurred at that timescale: CVs and IOIs should have increased most in Experiment 1, and error rates should have increased most in Experiment 2. Furthermore, comparisons of the first two experiments with Experiment 3 addressed whether disruption from combined alterations reflected a summation of disruption from each individual alteration. Analyses of mean error rates and mean IOIs for the normal feedback conditions did not reveal significant effects across experiments; however, mean CVs did differ across experiments, $F(2,91)=9.56, M S E=$ $.002, p<.01$. CVs were highest in Experiment $1(M=.089$ for Experiment $1 ; M=.045$ across Experiments 2 and 3), for which feedback alterations were predicted to yield the greatest disruption in timing; difference scores therefore provide a conservative measure of relative increases to $\mathrm{CVs}$ across experiments.

Figure 6 shows difference scores (altered - normal feedback) for CVs, mean IOIs, and error rates. Increases in CVs from altered feedback, shown in Figure 6A, differed across experiments, $F(2$, $44)=3.91, M S E=.001, p<.05$. As expected, the largest increase was found in Experiment 1, significantly exceeding difference scores from Experiment 2. Experiment 3 yielded intermediate difference scores that exceeded those of Experiment 2 (Tukey's HSD, $p<.05$ for all comparisons) but did not differ statistically from those of Experiment 1. Results for mean IOI, shown in Figure 6B, confirmed that Experiment 1 caused the greatest disruption to timing. Difference scores again varied across experiments, $F(2,44)=14.40, M S E=1,152, p<.01$, and post hoc tests indicated that the amount of slowing in Experiment 1 exceeded that in both other experiments, which did not differ from each other. Finally, altered feedback elicited greater increases in error rates in Experiment 2 than in other experiments, $F(2,44)=$ $5.54, M S E=.002, p<.01$, as shown in Figure 6C. Post hoc tests verified that difference scores in Experiment 2 exceeded those of 
A

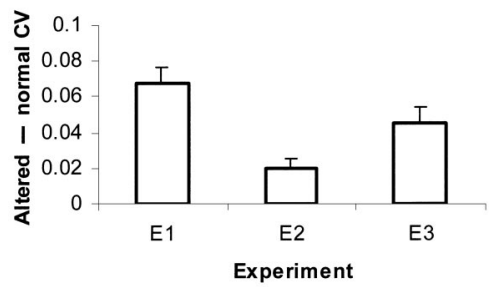

B

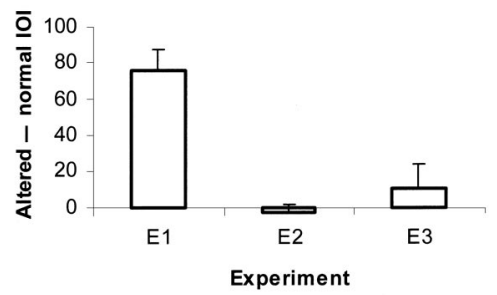

C

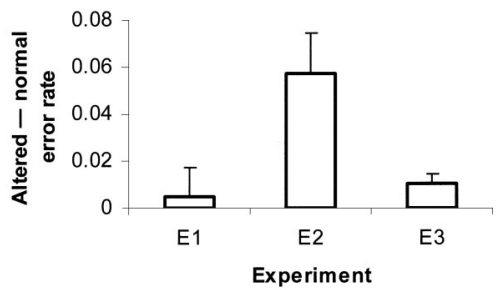

Figure 6. Difference scores (altered - normal) for effects of feedback alterations for different delay types in Experiments (Es) 1-3 for (A) mean coefficients of variation (CVs: standard deviation of interonset interval $[\mathrm{IOI}] /$ mean IOI), (B) mean IOIs, and (C) error rates. Altered feedback means are averaged across all binary meter conditions that featured no subdividing instructions. Error bars represent one between-participants standard error for difference scores.

Experiments 1 and 3, which did not differ from each other. It is important to note that combined alterations never yielded maximal disruption across experiments. This clearly rules out the possibility that disruption from combined alterations reflects summation of disruption from each alteration on its own.
A final issue of concern in the present study is whether disruption from altered feedback reflects the distinction between period and phase shifts or merely the temporal separation between actions and auditory feedback. Figure 7 addresses this issue by plotting feedback alterations from Experiments 1-3 along a single time continuum that combines period and phase shifts. Within each dependent measure, scores for individual participants and conditions were converted into standardized scores ( $Z$ scores) across experiments in order to facilitate comparisons across measures. In general, these data confirmed that qualitatively different patterns of disruption were found for measures of timing (CVs, IOIs) and accuracy (error rates) across different feedback alterations. $Z$ scores for $\mathrm{CV}$ s and IOIs were positively correlated with each other $(r=.69, p<.05)$, but correlations between $Z$ scores for error rates and each measure of timing were nonsignificant and negative (mean $r=-.19$ ). More specifically, maxima occurred in different locations for each measure, and positions of these maxima reflected different responses to phase versus period shifts rather than the temporal separation between actions and feedback. Period shifts (Experiment 2) constituted points of maximal error rates and minimal timing disruption. Timing variability and IOIs, on the other hand, increased most for phase shifts shorter than one cycle length (Experiment 1), although mean IOIs, unlike CVs, did not reach a maximum at .66. Combined alterations resulted in moderate levels of overall disruption.

\section{Discussion}

Experiment 3 demonstrated interactive effects of period and phase shifts on performance. Error rates elicited by one alteration type were qualified by the other alteration that was combined with it; a similar but nonsignificant pattern was found for CVs. The same statistical interaction was not found for IOIs; however, changes in IOI with amount of phase shift clearly differed from those found in Experiment 1. Further support for the interactive effects of phase and period shifts was found in comparisons across experiments: The degree of overall disruption from combined alterations did not reflect the sum of each alteration type on its own for each measure.

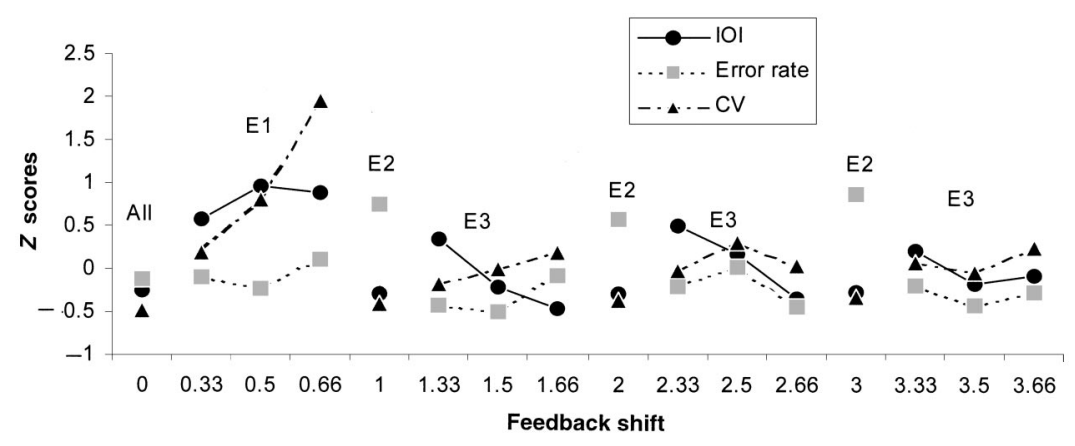

Figure 7. Effects of feedback manipulations across experiments plotted on a single time continuum for all dependent measures, in standardized units ( $Z$ scores). Conditions include binary meter conditions with no subdividing instructions. Values between 0 and 1 represent conditions from Experiment 1 (E1). Integer values represent conditions from Experiment 2 (E2). The remaining noninteger values represent conditions from Experiment 3 (E3). Normal feedback conditions (means and standard errors) are averaged across all experiments. $\mathrm{IOI}=$ interonset interval; $\mathrm{CV}=$ coefficient of variation. 


\section{Experiment 4}

A final experiment was carried out to confirm the comparisons across Experiments 1-3. Although the combined results of the first three experiments are compelling, there are nevertheless differences between experiments that may qualify the conclusions. Each participant in Experiment 4 experienced normal feedback, phase shifts, period shifts, and combined period and phase shifts in the same experimental session. The same stimuli materials were used as in Experiment 1, and the instructions and procedure were implemented as in Experiment 2.

\section{Method}

Participants. Eight adult pianists (mean age $=28$ years, range $=$ 19-45) from the San Antonio, Texas, community participated in this experiment for course credit in an introductory psychology class or payment. Pianists had 10 years of private piano training (range 4-16) and 21 years of experience playing the piano (range 10-36) on average. As in Experiment 3, one pianist had far fewer years of formal training (4) than other pianists (range $=7-16$ ) but was kept because of having many years of experience (36). Five pianists reported being right-handed, 2 reported being left-handed, and 1 was ambidextrous. Six participants were female, and 2 were male.

Conditions and design. The design fully crossed phase and period shifts of auditory feedback, including three levels of feedback phase shift $(0, .33$, and .66) and three levels of feedback period shift (0, Lag 1 , and Lag 2). Certain conditions were excluded (phase shifts of .5 and period shifts of Lag 3) in order to maintain a reasonable duration for the session. Participants performed each melody in all 9 feedback conditions, yielding 18 trials in a session. Trials were blocked and counterbalanced as in Experiment 3.

Apparatus. Pianists performed melodies on a Roland RD-700 weighted-key digital piano (an updated version of the piano used in the other experiments) and listened to auditory feedback over Sony MDR-7500 professional headphones at a listening level similar to that used in the other experiments.

\section{Results and Discussion}

A single ANOVA tested the effect of crossing phase shift $(0$, $.33, .66)$ and period shift (Lag 0, 1, 2). Following this analysis, correlations were calculated to compare the results with analogous conditions from the first three experiments (cf. Figure 7). Mean data for each dependent measure in Experiment 4 are shown in Figure 8 .

Mean error rates per trial are shown in Figure 8C. Error rates increased in the presence of feedback period shifts and were highest when these period shifts were not combined with phase shifts $(M=.0831 ; M$ across other conditions $=.035 ;$ range $=.02)$. The relatively low amount of disruption for combined alterations $(M=.045)$ further confirmed the nonadditive effects of feedback phase and period shifts. The ANOVA revealed a significant main effect of period shift, $F(2,14)=5.08, M S E=.001, p<.05$, and a Period Shift $\times$ Phase Shift interaction, $F(4,28)=3.24, M S E=$ $.001, p<.05$. The main effect of phase shift did not reach significance $(p=.103)$. The pattern of error rates across conditions correlated significantly with analogous conditions from Experiments $1-3(r=.77, p<.01)$.

Figure $8 \mathrm{~A}$ plots mean CVs across all combinations of feedback type. CVs increased with phase shifts when phase shifts were presented alone, as in Experiment 1, but they remained relatively
A

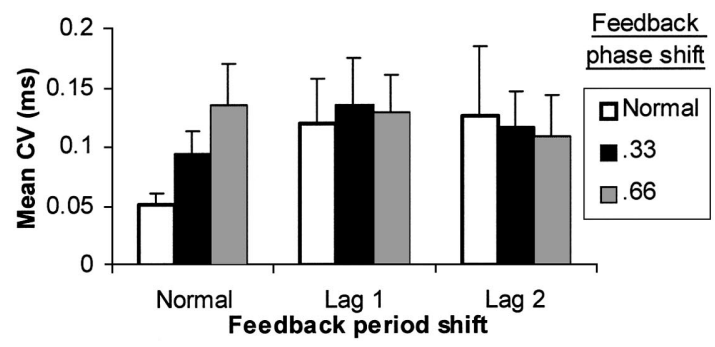

B

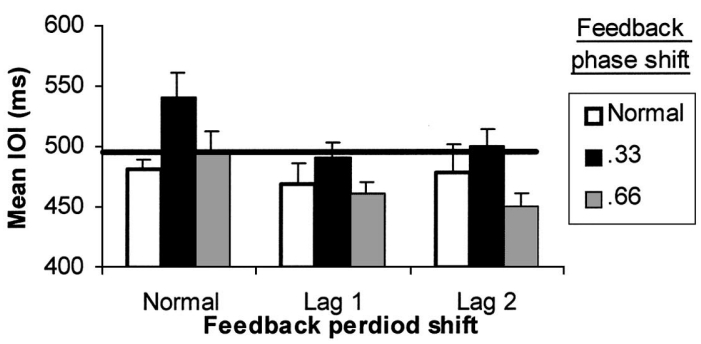

C

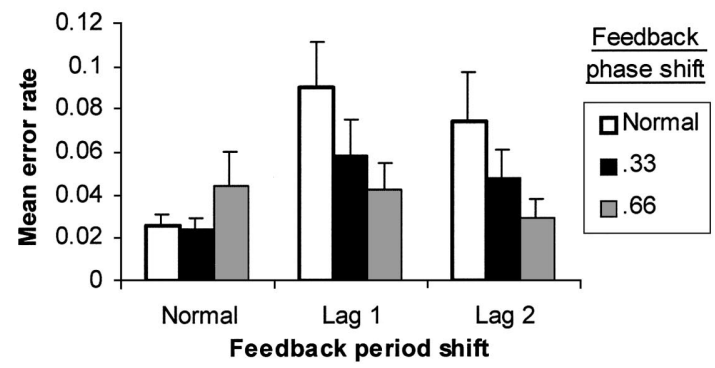

Figure 8. Disruptive effects of feedback alterations in Experiment 4 as indexed by (A) mean coefficients of variation (CVs: standard deviation of interonset interval [IOI]/mean IOI), (B) mean IOIs (black line corresponds to the $500 \mathrm{~ms}$ between metronome onsets), and (C) mean error rates. Error bars represent one between-participants standard error.

flat thereafter. However, the ANOVA did not reveal any significant effects. CV results were positively, but not significantly, correlated with analogous conditions from Experiments 1-3 $(r=$ $.45)$.

Finally, mean IOIs were examined and revealed a pattern similar to that found in Experiment 3. These data are shown in Figure 8B. Localized peaks in mean IOI appeared for each phase shift of .33, and this peak was largest when phase shifts of .33 were not combined with a period shift. The ANOVA revealed main effects of phase shift, $F(2,14)=11.75, M S E=1,014, p<.01$, and of period shift, $F(2,14)=7.67, M S E=1,031, p<.05$, but no interaction. It should be noted that the main effect of period shift reflected decreases in IOI (i.e., increases in production rate) and thus did not indicate disruption. Like error rates, mean IOIs were significantly correlated with analogous conditions from other experiments $(r=.76, p<.01)$.

\section{General Discussion}

Four experiments explored the effects of phase and period shifts of auditory feedback, relative to produced keypresses, in the performance of music. Three main conclusions arise from these 
experiments. First, both synchrony and correct contents of auditory feedback are necessary to maintain the fluency of production. Second, the kind of disruption to production brought about by altered feedback is related to the type of feedback alteration that a performer experiences. Phase shifts in the current study were most disruptive to produced timing, whereas period shifts were most disruptive to accuracy. It is interesting to note that combined alterations were not maximally disruptive. Third, some results from Experiment 2 suggest that discrepancies between sequence planning and feedback contents underlie disruption from period shifts.

The current data suggest that perception and action are coupled in a way that distinguishes hierarchical levels of temporal organization. The following discussion focuses first on the evidence for links between perception and action systems and then on the degree to which different timescales can be separated in both systems. Finally, a possible role of cognitive plans in the use of auditory feedback is considered.

\section{Links Between Perception and Action}

The primary purpose of this study was to explore the role of perception in action in the context of music performance. This study, like others, showed dependencies of production on auditory feedback. Some past studies have concluded that asynchrony between actions and auditory feedback influences production but that dissimilarity between expected and perceived feedback contents does not. The current data do not support the second conclusion, given that alterations of both feedback synchrony and feedback contents elicited disruption. The current manipulations differed from those used in past studies (e.g., Finney, 1997; Howell et al., 1983) in that both alterations resulted from calibrations of feedback timing at different levels of a single timing hierarchy via phase and period shifts. The finding of disruption from altered contents when these alterations result from period shifts, as opposed to randomized pitch contents (Finney, 1997), suggests that timing relationships do lie at the root of DAF disruption examined previously (cf. Finney \& Warren, 2002; Howell et al., 1983) but that the scope of relevant timing relationships may be broader and more differentiated than originally considered. Moreover, the absence of disruption from alterations of feedback contents in past research may reflect the fact that researchers did not fully take into account the possibility that melodic and rhythmic structure may jointly determine a sequence's temporal structure (Jones, 1976; Jones \& Pfordresher, 1997; Pfordresher, 2003).

This study, like others, examined how different magnitudes of a given alteration disrupt performance. Results in Experiment 1 for $\mathrm{CVs}$ and error rates converged with findings that disruption from asynchronous feedback increases as feedback onsets approach the next produced onset time (Finney \& Warren, 2002; Robinson, 1972). The results of these studies are inconsistent with the idea that a "critical interval" exists for delays around $200 \mathrm{~ms}$ (MacKay, 1987) and are more consistent with an account of disruption based on relative timing (e.g., Finney \& Warren, 2002; Howell et al., 1983). The .66 shift in Experiment 1 yielded an average absolute time lag of $345 \mathrm{~ms}$ for feedback onsets, with time lags ranging from 270 to $435 \mathrm{~ms}$. By contrast, the .5 and .33 shifts yielded absolute delay amounts closer to $200 \mathrm{~ms}$ (50\% delay: $M=264 \mathrm{~ms}$, range $=196-329 \mathrm{~ms} ; 33 \%$ delay: $M=167 \mathrm{~ms}$, range $=129-204$ $\mathrm{ms})$, but they elicited lower disruption. The results of Experiment 2 differed from those of other studies in that disruption did not differ across period shifts overall, which suggests that the magnitude of alterations is perceived differently for phase versus period shifts. Finally, the fact that the monotonic increases in disruption with phase shift amount, found for CVs and error rates in Experiment 1 , changed when these phase manipulations were combined with period shifts (alterations to contents) further demonstrates that onset synchrony alone cannot account for disruption from altered feedback, as would be predicted by the rhythmic displacement hypothesis of Howell (Howell et al., 1983). Similarly, Finney (1997) found lower overall disruption from DAF when pitches were randomized than when DAF presented veridical pitch contents.

Some findings in the present study that are difficult to explain involve discrepancies between mean IOIs and CVs across different phase and period shifts. In particular, mean IOIs in Experiments 3 and 4 were maximal for phase shifts of .33 (for which the absolute time lag was around $200 \mathrm{~ms}$ ) and reached an asymptote for the same phase shift in Experiment 1. Both results are consistent with the critical interval hypothesis. Moreover, no interaction of phase and period shifts emerged for mean IOIs in Experiments 3 or 4, in contrast with error rates and CVs in Experiment 3. It may be that the guidance of production rate is less sensitive to perceptionaction relationships at higher levels (e.g., sequencing) than the control of timing regularity. If so, this may explain why past studies using mean IOI have demonstrated that producers are insensitive to feedback contents (Howell et al., 1983). However, it is also important to note that shorter mean IOIs were produced for combined alterations than for phase shifts, which points to nonadditivity of phase and period shifts for this measure. Moreover, despite the presence of some differences between CVs and mean IOIs, both measures were highly correlated across phase and period shifts for Experiments 1-3, and global patterns of disruption were highly consistent across both measures.

The connection between perception and production demonstrated here is consistent with findings in the music cognition literature demonstrating similar expectations for expressive timing deviations in listeners and performers (Drake, 1993; Repp, 1995, 1998a, 1998b, 1999a), as well as findings that both performers and listeners separate lower and higher time scales in music (Drake, 1998; Jones \& Boltz, 1989; Penel \& Drake, 1998, 2000). Such findings, which derived from separate tests of perception and production, may reflect the kind of coupling suggested by the current data. It is possible that the perception of expressivity in music performances stems from implied motor gestures that arise in the time structure of performance (see Epstein, 1995; Todd, 1992, 1995, for similar theoretical perspectives).

\section{Sequencing and Timing at Separable Time Scales}

Another goal of this study was to explore the role of feedback alterations across different timescales of sequence organization by varying relationships between actions and auditory feedback in terms of period and phase shifts. Perhaps the strongest new result from the current study was the double dissociation found in the effects of these alterations: Phase shifts mainly disrupted timing, whereas period shifts mainly disrupted accuracy. These results suggest that relationships between action and perception are me- 
diated by a common, hierarchical representation in the spirit of common-coding theory (Hommel et al., 2001; Prinz, 1997). The present results further suggest that functional relationships may be distinguished according to timescales of sequence organization. In the terms used by common-coding theory, timing and sequencing may be two features that distinguish events in the context of a melody, and perception and action share a common representation of these features. That is, the timescale on which the alteration occurs may interfere with the commensurate timescale in production.

The dissociation found in the current study also bears on the theoretical distinction between sequencing and timing. In general, the current results support the separation of these different hierarchical levels of sequence organization (MacKay, 1987; Martin, 1972; Palmer, 1997) and are inconsistent with models in which production is guided only by timing or only by sequencing (e.g., Rosenbaum et al., 1983; Rumelhart \& Norman, 1981). However, alterations to both characteristics led to interactions, and in no case was disruption of one characteristic absent (except mean IOI), contrary to predictions. Timing and sequencing therefore appear to be distinct yet interactive. This assumption has been instantiated in other models of sequence production or perception (e.g., Large \& Jones, 1999; Palmer \& Pfordresher, in press), although no existing model appears to completely account for the present effects.

\section{The Role of Cognitive Planning}

A final goal of this work was to establish whether altered feedback interferes with planning of actions. Plans, as defined here, involve the use of hierarchical cognitive representations to guide production and perception. Manipulations of mental subdividing in Experiment 1 attempted to influence how performers conceptualized performance at the level of timing between onsets. Manipulations of meter in Experiment 2 attempted to influence how performers conceptualized performance at the level of sequencing among series of events. The logic behind each manipulation was that certain planning strategies may be better suited to certain feedback alterations if feedback alterations cause disruption via cognitive plans. Manipulations of planning yielded mixed results. Planning of timing in Experiment 1 did not modulate disruption from phase shifts, whereas planning of sequencing in Experiment 2 did modulate disruption from period shifts, though not for all performers.

Effects of subdividing in Experiment 1 provided a general facilitation of performance but did not reduce disruption at predicted phase shifts (cf. Pfordresher \& Palmer, 2002; Treffner \& Turvey, 1993; Vos \& Helsper, 1992). Experiment 1's results are instead consistent with research in which mental counting has produced a general improvement to duration estimation (Gillard \& Martin, 1940; Hicks \& Allen, 1979; Wearden, 1991) and discrimination (Grondin, Meilleur-Wells, \& Lachance, 1999) and may reflect greater attentional allocation to time (cf. Brown \& Boltz, 2002). The failure to connect planning with disruption from phase shifts converges with other claims that asynchronies between actions and perceived events have their effects at an early stage of processing that does not involve cognitive plans (Finney \& Warren, 2002; Howell et al., 1983; Repp, 2000, 2001, 2002). However, there are a number of alternative reasons why subdividing failed to support the predicted connection between planning and disruption from phase shifts. It may be that the complexity of produced sequences in Experiment 1 (relative to arpeggios used by Pfordresher \& Palmer, 2002) imposed cognitive demands that prevented participants from benefitting from such timing relationships (cf. Pellecchia \& Turvey, 2001). Also, it is possible that performers were not able to subdivide appropriately, given the rapid rate of $250 \mathrm{~ms}$ or $167 \mathrm{~ms}$ per subinterval (Friberg \& Sundberg, 1995; Hibi, 1983; London, 2002; Peters, 1989). Some support for this second possibility emerged from responses to postexperiment questionnaires, in which participants indicated that they maintained an intermediate level of accuracy in mental subdividing.

Manipulations of meter in Experiment 2 suggest that planning can modulate disruption from period shifts, although the effect was found for only about half of the participants. How should differences among participants be interpreted? One possibility is that performers at different skill levels use planning and auditory feedback differently. For instance, three participants who showed no disruption at all from period-shifted feedback reported performing the piano the most hours per week and having the most years of experience in comparison with other participants. This relationship suggests that these participants did not have to confirm the execution of planned events through auditory feedback, which is consistent with evidence for enhanced domain-specific memory in experts (W. G. Chase \& Simon, 1973; Ericsson \& Kintsch, 1995; Sloboda, 1984; Vincente \& Wang, 1998). Conversely, those participants who were disrupted but did not show the predicted pattern of results reported playing the piano the fewest hours per week, had the least experience, and also produced the highest overall error rates. These musicians may not have made use of abstract structures such as musical meter to aid memory, relying instead on the veridicality of auditory feedback for confirmation of each individual produced event (cf. Finney \& Palmer, 2003; Fitts \& Posner, 1967). The predicted interaction between meter and disruption may therefore reflect an intermediate level of skill at which abstract structures guide memory processes while feedback is also used to monitor the outcomes of production.

Although manipulations of explicit planning proved ambiguous, the present results overall do suggest some contribution of planning. That is, it is likely that these effects stem from interference between planned actions and perceived events when the perceived outcomes of actions differ from expected outcomes (those associated with produced keypresses). Recent research on choice response tasks has revealed that perceptual events may interfere with planned actions and that interference increases when the perceived event suggests a response incompatible with the planned responses (Müsseler, 1999). In this spirit, results of the current Experiment 2 are best described as resulting from conflicts between expected (i.e., planned) and perceived (i.e., feedback) contents. Period shifts ultimately resulted in the contents of feedback not being mapped to its usual spatial location but instead to locations associated with previously executed actions. Additional evidence linking feedback with cognition has arisen in recent studies of category learning (Maddox, 2002).

The results from Experiment 3 may also be interpreted in the context of planning, although planning was not explicitly manipulated in that experiment. Disruption was reduced in general for the combined alterations in Experiment 3 relative to the other experiments. These results suggest that increasing dissimilarity between the expected outcomes of actions and feedback can even- 
tually result in the dissociation of feedback from production. The fact that reduced interference was found for feedback alterations that combined phase and period shifts provides some further support for the idea that sequencing and timing relationships between action and perception interact. That is, when feedback is dissociated from actions in terms of both phase and period, disruption is reduced in comparison with situations in which feedback is dissociated in terms of one but not the other. This nonlinear relationship between similarity and disruption may also account for why randomizations of feedback contents do not disrupt production (e.g. Finney, 1997): Randomized pitches form an auditory pattern that is highly dissimilar from the planned sequence of events.

This interpretation helps account for an unusual result from Experiment 3: The fact that the Lag $2+.5$ shift condition yielded highest disruption in errors and CVs. This finding was unexpected, given the relatively low disruption for these conditions in Experiments 1 and 2 . It is possible that the Lag $2+.5$ shift condition may have disrupted most in Experiment 3 because it more strongly matched the current status of planning than other delay conditions. The lag of 2 presents a metrically similar event (cf. Experiment 2), and a phase shift of .5 is relatively stable rhythmically (cf. Pfordresher \& Palmer, 2002). Further support for this interpretation comes from Müsseler (1999), who has suggested that similarity between perception and action may facilitate planned actions when both event are synchronous, whereas such similarity may interfere with planned actions when actions and perceptual events are asynchronous.

Although the current study has focused on musical behavior, some of the findings may generalize to the linguistic domain. Past results from tasks with DAF corroborate other evidence that linguistic and musical structure are processed similarly (Besson \& Schön, 2001; Patel, 1998). One limitation of the present research is that it examined only auditory feedback, whereas a variety of feedback sources contribute to the performance of music, including vision, touch, (Gabrielsson, 1999; Pressing, 1988), and proprioception (Todd, 1999). It is likely that patterns of disruption differ as a function of which and how many sources of feedback are altered (cf. Aschersleben \& Prinz, 1995, 1997), but such manipulations were beyond the scope of the current study.

In conclusion, the results of these experiments suggest that salient timing relationships between the production of complex sequences and auditory feedback are broader and more differentiated than was once thought. Disruption from feedback shifts spanned several events, and patterns of disruption revealed a dissociation between phase and period shifts. It appears that the perception-action system is coupled in a way that distinguishes these timescales (cf. Jones \& Boltz, 1989; Large \& Jones, 1999; Palmer \& Pfordresher, in press). One theoretical possibility arises from the idea that perception and action share a common representational domain (Hommel et al., 2001; Prinz, 1997), hence feedback alterations at a given timescale disrupt production at that same timescale.

\section{References}

Aschersleben, G., \& Prinz, W. (1995). Synchronizing actions with events: The role of sensory information. Perception \& Psychophysics, 57, 305317 .
Aschersleben, G., \& Prinz, W. (1997). Delayed auditory feedback in synchronization. Journal of Motor Behavior, 29, 35-46.

Besson, M., \& Schön, D. (2001). Comparison between language and music. In R. J. Zatorre \& I. Peretz (Eds.), The biological foundations of music: Annals of the New York Academy of Sciences (Vol. 930, pp. 232-258). New York: New York Academy of Sciences.

Black, J. W. (1951). The effect of delayed side-tone upon vocal rate and intensity. Journal of Speech and Hearing Disorders, 16, 56-60.

Bock, K. (1995). Sentence production: From mind to mouth. In J. L. Milles \& P. D. Eimas (Eds.), Speech, language, and communication: Handbook of perception and cognition (Vol. 11, 2nd ed., pp. 181-216). San Diego, CA: Academic Press.

Bock, K. (1999). Language production. In R. A. Wilson \& F. C. Keil (Eds.), The MIT encyclopedia of the cognitive sciences (pp. 453-456). Cambridge, MA: MIT Press.

Brown, S. W., \& Boltz, M. G. (2002). Attentional processes in time perception: Effects of mental workload and event structure. Journal of Experimental Psychology: Human Perception and Performance, 28, $600-615$.

Chase, R. A. (1965). An information-flow model of the organization of motor activity: I. Transduction, transmission and central control of sensory information. Journal of Nervous and Mental Disease, 140, $239-251$.

Chase, R. A., Harvey, S., Standfast, S., Rapin, I., \& Sutton, S. (1961). Studies on sensory feedback: I. Effect of delayed auditory feedback on speech and keytapping. Quarterly Journal of Experimental Psychology, $13,141-152$.

Chase, W. G., \& Simon, H. A. (1973). The mind's eye in chess. In W. G. Chase (Ed.), Visual information processing (pp. 215-281). New York: Academic Press.

Collard, R., \& Povel, D. J. (1982). Theory of serial pattern production: Tree traversals. Psychological Review, 89, 693-707.

Cooper, G., \& Meyer, L. B. (1960). The rhythmic structure of music. Chicago: University of Chicago Press.

Dell, G. S. (1986). A spreading-activation theory of retrieval in sentence production. Psychological Review, 93, 283-321.

Dell, G. S., Burger, L. K., \& Svec, W. R. (1997). Language production and serial order: A functional analysis and a model. Psychological Review, 104, 123-147.

Drake, C. (1993). Perceptual and performed accents in musical sequences. Bulletin of the Psychonomic Society, 31, 107-110.

Drake, C. (1998). Psychological processes involved in the temporal organization of complex auditory sequences: Universal and acquired processes. Music Perception, 16, 11-26.

Drake, C., \& Palmer, C. (1993). Accent structures in music performance. Music Perception, 10, 343-378.

Epstein, D. (1995). Shaping time: Music, the brain, and performance. New York: Schirmer.

Ericsson, K. A., \& Kintsch, W. (1995). Long-term working memory. Psychological Review, 102, 211-245.

Fairbanks, G. (1954). Systematic research in experimental phonetics: 1. A theory of the speech mechanism as a servosystem. Journal of Speech and Hearing Disorders, 19, 133-139.

Fairbanks, G., \& Guttman, N. (1958). Effects of delayed auditory feedback upon articulation. Journal of Speech and Hearing Research, 1, 333-346.

Finney, S. A. (1997). Auditory feedback and musical keyboard performance. Music Perception, 15, 153-174.

Finney, S. A. (2001). FTAP: A Linux-based program for tapping and music experiments. Behavior Research Methods, Instruments, \& Computers, $33,65-72$

Finney, S. A., \& Palmer, C. (2003). Auditory feedback and memory for music performance: Sound evidence for an encoding effect. Memory \& Cognition, 31, 51-64.

Finney, S. A., \& Warren, W. H. (2002). Delayed auditory feedback and 
rhythmic tapping: Evidence for a critical interval shift. Perception \& Psychophysics, 64, 896-908.

Fitts, P. M., \& Posner, M. I. (1967). Human performance. Belmont, CA: Brooks/Cole

Friberg, A., \& Sundberg, J. (1995). Time discrimination in a monotonic, isochronous sequence. Journal of the Acoustical Society of America, 98, $2524-2530$

Fromkin, V. A. (Ed.). (1973). Speech errors as linguistic evidence. The Hague, the Netherlands: Mouton.

Gabrielsson, A. (1999). The performance of music. In D. Deutsch (Ed.), The psychology of music (pp. 501-602). San Diego, CA: Academic Press.

Garrett, M. F. (1980). Levels of processing in sentence production. In B. Butterworth (Ed.), Language production: Speech and talk (pp. 177220). London: Academic Press.

Gates, A., \& Bradshaw, J. L. (1974). Effects of auditory feedback on a musical performance task. Perception \& Psychophysics, 16, 105-109.

Gates, A., Bradshaw, J., \& Nettleton, N. (1974). Effect of different delayed auditory feedback intervals on a music performance task. Perception \& Psychophysics, 15, 21-25.

Gilliard, A. R., \& Martin, R. (1940). Some factors in estimating short time intervals. Journal of Experimental Psychology, 27, 243-255.

Grondin, S., Meilleur-Wells, G., \& Lachance, R. (1999). When to start explicit counting in a time-intervals discrimination task: A critical point in the timing process of humans. Journal of Experimental Psychology: Human Perception and Performance, 25, 993-1004.

Hibi, S. (1983). Rhythm perception in repetitive sound sequence. Journal of the Acoustical Society of Japan, 4, 83-95.

Hicks, R. E., \& Allen, D. A. (1979). Counting eliminates the repetition effect in judgments of temporal duration. Acta Psychologica, 43, 361366

Hommel, B., Müsseler, J., Aschersleben, G., \& Prinz, W. (2001). The theory of event coding (TEC): A framework for perception and action planning. Behavioral and Brain Sciences, 24, 849-937.

Howell, P. (1983). The effect of delaying auditory feedback of selected components of the speech signal. Perception \& Psychophysics, 34, 387-396.

Howell, P., \& Archer, A. (1984). Susceptibility to the effects of delayed auditory feedback. Perception \& Psychophysics, 36, 296-302.

Howell, P., \& Powell, D. J. (1987). Delayed auditory feedback with delayed sounds varying in duration. Perception \& Psychophysics, 42, $166-172$.

Howell, P., Powell, D. J., \& Khan, I. (1983). Amplitude contour of the delayed signal and interference in delayed auditory feedback tasks. Journal of Experimental Psychology: Human Perception and Performance, 9, 772-784.

Jagacinski, R. J., Marshburn, E., Klapp, S. T., \& Jones, M. R. (1988). Tests of parallel versus integrated structure in polyrhythmic tapping. Journal of Motor Behavior, 20, 416-442.

Jones, M. R. (1976). Time, our lost dimension: Toward a new theory of perception, attention, and memory. Psychological Review, 83, 323-355.

Jones, M. R., \& Boltz, M. (1989). Dynamic attending and responses to time. Psychological Review, 96, 459-491.

Jones, M. R., \& Pfordresher, P. Q. (1997). Tracking melodic events using joint accent structure. Canadian Journal of Experimental Psychology, 51, 271-291

Kelso, J. A. S. (1995). Dynamic patterns. Cambridge, MA: MIT Press.

Keppel, G. (1991). Design and analysis: A researcher's handbook. Upper Saddle River, NJ: Prentice-Hall.

Kirk, R. E. (1995). Experimental design: Procedures for the behavioral sciences (3rd ed.). Pacific Grove, CA: Brooks/Cole.

Large, E. W. (2000). On synchronizing movements to music. Human Movement Science, 19, 527-566.
Large, E. W., Fink, P., \& Kelso, J. A. S. (2002). Tracking simple and complex sequences. Psychological Research, 66, 3-17.

Large, E. W., \& Jones, M. R. (1999). The dynamics of attending: How people track time-varying events. Psychological Review, 106, 119-159.

Large, E. W., \& Palmer, C. (2002). Perceiving temporal regularity in music. Cognitive Science, 26, 1-37.

Lashley, K. (1951). The problem of serial order in behavior. In L. A. Jeffress (Ed.), Cerebral mechanisms in behavior (pp. 112-136). New York: Wiley.

Lee, B. S. (1950). Effects of delayed speech feedback. Journal of the Acoustical Society of America, 22, 824-826.

Lee, B. S. (1951). Artificial stutter. Journal of Speech and Hearing Disorders, 16, 53-55.

Lerdahl, F., \& Jackendoff, R. (1983). A generative theory of tonal music. Cambridge, MA: MIT Press.

Liberman, M., \& Prince, A. (1977). On stress and linguistic rhythm. Linguistic Inquiry, 8, 249-336.

London, J. (2002). Cognitive constraints on metric systems: Some observations and hypotheses. Music Perception, 19, 529-530.

MacKay, D. G. (1968). Metamorphosis of a critical interval: Age-linked changes in the delay in auditory feedback that produces maximal disruption of speech. Journal of the Acoustical Society of America, 43, 811-821.

MacKay, D. G. (1970). How does language familiarity influence stuttering under delayed auditory feedback? Perceptual and Motor Skills, 30, 655-669.

MacKay, D. G. (1987). The organization of perception and action. New York: Springer-Verlag.

Maddox, W. T. (2002). Feedback and response characteristic effects on rule-based and information integration category learning. Abstracts of the Psychonomic Society, 7, 36.

Martin, J. G. (1972). Rhythmic (hierarchical) versus serial structure in speech and other behavior. Psychological Review, 79, 485-509.

Meyer, R. K., \& Palmer, C. (2002). Rate and tactus effects on timing and serial order in music performance. Manuscript submitted for publication.

Müller, K., Aschersleben, G., Esser, K. H., \& Müsseler, J. (2000). Effects of delayed auditory feedback on speech: Just a problem of displaced rhythm? In P. Desain \& L. Windsor (Eds.), Rhythm perception and production (pp. 249-262). Lisse, the Netherlands: Swets \& Zeitlinger.

Müsseler, J. (1999). How independent from action control is perception? An event coding account for more equally-ranked crosstalks. In G. Aschersleben, T. Bachmann, \& J. Müsseler (Eds.), Cognitive contributions to the perception of spatial and temporal events (pp. 121-148). Amsterdam: Elsevier

Norman, D. (1981). Categorization of action slips. Psychological Review, 88, 1-15.

Palmer, C. (1997). Music performance. Annual Review of Psychology, 48, $115-138$.

Palmer, C., \& Drake, C. (1997). Monitoring and planning capacities in the acquisition of music performance skills. Canadian Journal of Experimental Psychology, 51, 369-384.

Palmer, C., \& Krumhansl, C. L. (1990). Mental representations for musical meter. Journal of Experimental Psychology: Human Perception and Performance, 16, 728-741.

Palmer, C., \& Pfordresher, P. Q. (in press). Incremental planning in sequence production. Psychological Review.

Palmer, C., \& Schendel, Z. A. (2002). Working memory constraints in sequence production. Abstracts of the Psychonomic Society, 7, 30.

Palmer, C., \& van de Sande, C. (1993). Units of knowledge in music performance. Journal of Experimental Psychology: Learning, Memory, and Cognition, 19, 457-470.

Palmer, C., \& van de Sande, C. (1995). Range of planning in skilled music 
performance. Journal of Experimental Psychology: Human Perception and Performance, 21, 947-962.

Patel, A. D. (1998). Syntactic processing in language and music: Different cognitive operations, similar neural resources? Music Perception, 16, $27-42$.

Pellecchia, G. L., \& Turvey, M. T. (2001). Cognitive activity shifts the attractors of bimanual rhythmic coordination. Journal of Motor Behavior, 33, 9-15.

Penel, A., \& Drake, C. (1998). Sources of timing variations in music performance: A psychological segmentation model. Psychological Research, 61, 12-32.

Penel, A., \& Drake, C. (2000). Rhythm in music performance and perceived structure. In P. Desain \& L. Windsor (Eds.), Rhythm perception and production (pp. 225-232). Lisse, the Netherlands: Swets \& Zeitlinger.

Peters, M. (1989). The relationship between variability of intertap intervals and interval duration. Psychological Research, 51, 38-42.

Pfordresher, P. Q. (2003). The role of melodic and rhythmic accents in musical structure. Music Perception, 20, 431-464.

Pfordresher, P. Q., \& Palmer, C. (2002). Effects of delayed auditory feedback on timing of music performance. Psychological Research, 66, 71-79.

Pikovsky, A., Rosenblum, M., \& Kurths, J. (2001). Synchronization: A universal concept in nonlinear sciences. Cambridge, England: Cambridge University Press.

Pitt, M. A., \& Samuel, A. G. (1990). The use of rhythm in attending to speech. Journal of Experimental Psychology: Human Perception and Performance, 16, 564-573.

Povel, D. J. (1981). Internal representation of simple temporal patterns. Journal of Experimental Psychology: Human Perception and Performance, 7, 3-18.

Povel, D. J., \& Essens, P. (1985). Perception of temporal patterns. Music Perception, 2, 411-440

Pressing, J. (1988). Improvisation: Methods and models. In J. A. Sloboda (Ed.), Generative processes in music: The psychology of performance, improvisation, and composition (pp. 129-178). Oxford, England: Clarendon Press.

Pressing, J. (1999). The referential dynamics of cognition and action. Psychological Review, 106, 714-747.

Prinz, W. (1997). Perception and action planning. European Journal of Cognitive Psychology, 9, 129-154.

Repp, B. H. (1995). Detectability of duration and intensity increments in melody tones: A partial connection between music perception and performance. Perception \& Psychophysics, 57, 1217-1232.

Repp, B. H. (1998a). Obligatory "expectations" of expressive timing induced by perception of musical structure. Psychological Research, 61, 33-43.

Repp, B. H. (1998b). Variations on a theme by Chopin: Relations between perception and production of timing in music. Journal of Experimental Psychology: Human Perception and Performance, 24, 791-811.

Repp, B. H. (1999a). Detecting deviations from metronomic timing in music: Effects of perceptual structure on the mental timekeeper. Perception \& Psychophysics, 61, 529-548.

Repp, B. H. (1999b). Effects of auditory feedback deprivation on expressive piano performance. Music Perception, 16, 409-438.

Repp, B. H. (2000). Compensation for subliminal timing perturbations in perceptual-motor synchronization. Psychological Research, 63, 106128.

Repp, B. H. (2001). Phase correction, phase resetting, and phase shifts after subliminal timing perturbations in sensorimotor synchronization. Journal of Experimental Psychology: Human Perception and Performance, 27, 600-621.

Repp, B. H. (2002). Automaticity and voluntary control of phase correction following event onset shifts in sensorimotor synchronization. Journal of Experimental Psychology: Human Perception and Performance, 28, $410-430$.

Repp, B. H. (2003). Phase attraction in sensorimotor synchronization with auditory sequences: Effects of single and periodic distractors on synchronization accuracy. Journal of Experimental Psychology: Human Perception and Performance, 29, 290-309.

Robinson, G. M. (1972). The delayed auditory feedback effect is a function of speech rate. Journal of Experimental Psychology, 95, 1-5.

Rosenbaum, D. A., Kenny, S. B., \& Derr, M. A. (1983). Hierarchical control of rapid movement sequences. Journal of Experimental Psychology: Human Perception and Performance, 9, 86-102.

Rumelhart, D. E., \& Norman, D. A. (1981). Simulating a skilled typist: A study of skilled cognitive-motor performance. Cognitive Science, 6 , $1-36$.

Semjen, A., \& Ivry, R. B. (2001). The coupled oscillator model of betweenhand coordination in alternate-hand tapping: A reappraisal. Journal of Experimental Psychology: Human Perception and Performance, 27, 251-265.

Semjen, A., Vorberg, D., \& Schulze, H. (1998). Getting synchronized with the metronome: Comparisons between phase and period correction. Psychological Research, 61, 44-55.

Sloboda, J. A. (1984). Experimental studies of musical reading: A review. Music Perception, 2, 222-236.

Thaut, M. H., Miller, R. A., \& Schauer, L. M. (1998). Multiple synchronization strategies in rhythmic sensorimotor tasks: Phase vs. period correction. Biological Cybernetics, 79, 241-250.

Todd, N. P. M. (1992). The dynamics of dynamics: A model of musical expression. Journal of the Acoustical Society of America, 91, 35403550.

Todd, N. P. M. (1995). The kinematics of musical expression. Journal of the Acoustical Society of America, 97, 1940-1949.

Todd, N. P. M. (1999). Motion in music: A neurobiological perspective. Music Perception, 17, 115-126.

Treffner, P. J., \& Turvey, M. T. (1993). Resonance constraints on rhythmic movement. Journal of Experimental Psychology: Human Perception and Performance, 19, 1221-1237.

Vincente, K. J., \& Wang, J. H. (1998). An ecological theory of expertise effects in memory recall. Psychological Review, 105, 33-56.

Vos, P. G., \& Helsper, E. L. (1992). Tracking simple rhythms: On-beat versus off-beat performance. In F. Macar, V. Pouthas, \& W. J. Friedman (Eds.) Time, action and cognition: Towards bridging the gap (pp. 287-299). Dordrecht, the Netherlands: Kluwer.

Wearden, J. H. (1991). Do humans possess an internal clock with scalar timing properties? Learning \& Motivation, 22, 59-83.

Received February 27, 2002

Revision received April 4, 2003 Accepted April 13, 2003 